\title{
Development of a Nonequilibrium Radiative Heating Prediction Method for Coupled Flowfield Solutions
}

\author{
Lin C. Hartung* \\ NASA Langley Research Center, Hampton, VA 23665-5225
}

\begin{abstract}
A method for predicting radiative heating and coupling effects in nonequilibrium flowfields has been developed. The method resolves atomic lines with a minimum number of spectral points, and treats molecular radiation using the smeared band approximation. To further minimize computational time, the calculation is performed on an optimized spectrum, which is computed for each flow condition to enhance spectral resolution. Additional time savings are obtained by performing the radiation calculation on a subgrid optimally selected for accuracy. Representative results from the new method are compared to previous work to demonstrate that the speedup does not cause a loss of accuracy and is sufficient to make coupled solutions practical. The method is found to be a useful tool for studies of nonequilibrium flows.
\end{abstract}

\section{Nomenclature}

$A_{u l} \quad$ transition probability $(/ \mathrm{sec})$

\footnotetext{
*Aerospace Engineer, Aerothermodynamics Branch, Space Systems Division, NASA Langley Research Center, Member AIAA.

Received April 22, 1991; presented as Paper 91-1406 at the AIAA 26th Thermophysics Conference, Honolulu, HI, June 24-26, 1991; revision received Oct. 10, 1991; accepted for publication Oct. 14, 1991. Copyright (c) 1991 by the American Institute of Aeronautics and Astronautics, Inc. No copyright is asserted in the United States under Title 17, U.S. Code. The U.S Government has a royalty-free license to exercise all rights under the copyright claimed herein for Governmental purposes. All other rights are reserved by the copyright owner.
} 


\begin{tabular}{|c|c|}
\hline$b$ & normalized line shape (sec) \\
\hline$B_{v}, B_{e}$ & rotational constants $(\mathrm{eV})$ \\
\hline$c$ & speed of light $(\mathrm{cm} / \mathrm{sec})$ \\
\hline$C_{i}$ & concentration of species $i$ \\
\hline$D_{v}, D_{e}$ & 2nd rotational constants $(\mathrm{eV})$ \\
\hline$D_{e l}$ & matrix element (statcoul-cm) \\
\hline$e$ & electron charge (statcoul) \\
\hline$E$ & emission $\left(\mathrm{W} / \mathrm{cm}^{3}\right)$ \\
\hline$E_{n}$ & energy or energy level $(\mathrm{eV})$ \\
\hline$f$ & distribution function \\
\hline$f_{l u}$ & oscillator strength for line transitions \\
\hline$F$ & normalized line shape \\
\hline$g$ & degeneracy \\
\hline$h$ & Planck's constant (eV-sec or erg-sec) \\
\hline$I$ & ionization potential of ground state $(\mathrm{eV})$ \\
\hline$I_{\nu}$ & radiative intensity ( W/ $\mathrm{cm}^{2}-\mathrm{sec}^{-1}$-ster) \\
\hline$j_{\nu}^{e}$ & emission coefficient $\left(\mathrm{W} / \mathrm{cm}^{3}-\mathrm{sec}^{-1}\right.$-ster $)$ \\
\hline$J^{e}$ & radiative emission in a line \\
\hline$J$ & rotational quantum number \\
\hline$k$ & Boltzmann's constant $(\mathrm{eV} / \mathrm{K}$ or erg/K) \\
\hline$m$ & $\operatorname{mass}(g)$ \\
\hline$n$ & principal quantum number \\
\hline$n_{i}$ & ionization limit \\
\hline$n^{*}$ & photoionization threshold level \\
\hline$N$ & number density $\left(\mathrm{cm}^{-3}\right)$ \\
\hline$p_{J^{u} J^{l}}$ & rotational transition probability \\
\hline$q_{R}$ & radiative flux $\left(\mathrm{W} / \mathrm{cm}^{2}\right)$ \\
\hline
\end{tabular}




$\begin{array}{ll}q_{v^{u} v^{l}} & \text { Franck-Condon factor } \\ Q & \text { partition function } \\ T & \text { temperature }(\mathrm{K}) \\ \mathrm{V} & \text { electron speed }(\mathrm{cm} / \mathrm{sec}) \\ v & \text { vibrational quantum number } \\ W & \text { weighting factor for subgrid } \\ Z & \text { ion charge number } \\ Z_{k} & \text { subgrid weighting function } \\ \alpha & \text { correction to rotational constant }(\mathrm{eV}) \\ \eta & \text { wall normal }(\mathrm{cm}) \\ \kappa_{\nu} & \text { absorption coefficient }(/ \mathrm{cm}) \\ \lambda & \text { wavelength }(\mathrm{cm}) \\ \nu & \text { frequency }(/ \mathrm{sec}) \\ \sigma & \text { cross section }\left(\mathrm{cm}{ }^{2}\right) \\ \omega_{e} & \text { spectroscopic constant }(\mathrm{eV})\end{array}$

\section{Subscripts}

$\begin{array}{ll}A, B & \text { lower and upper electronic states } \\ c & \text { capture } \\ e & \text { electron or electronic } \\ e x & \text { excitation } \\ l & \text { lower } \\ n & \text { energy level } n \\ r & \text { rotational } \\ t & \text { translational } \\ u & \text { upper }\end{array}$


$+\quad$ ion

\section{Introduction}

Proposed aerobraking vehicles will experience significant periods of nonequilibrium flow

in the upper layers of the atmosphere. In order to support studies of these flowfields a nonequilibrium radiation model has been developed. This model is designed to take temperatures and species concentrations from a nonequilibrium flowfield solver, such as the Langley Aerothermodynamic Upwind Relaxation Algorithm (LAURA) CFD code of Gnoffo, ${ }^{1,2}$ and predict the radiative heat flux to the vehicle surface. The new method is called the Langley Optimized RAdiative Nonequilibrium (LORAN) code. It is intended to fill a void currently existing between fast but highly approximate methods, and detailed but computationally costly methods.

Preliminary results from this method were presented in Ref. 3. The present paper will describe the method, discuss issues that arose during its development, and present a few additional results. Complete details of the method are available in a separate reference. ${ }^{4}$

\section{Theory}

Radiation emission and absorption in a gas result from transitions between energy levels. The absorption coefficient can be written as the product of the population of an energy level and its radiation absorption cross section summed over all the energy levels available:

$$
\kappa_{\nu}=\sum_{\text {energy levels } \mathrm{n}} N_{n} \sigma_{\nu n}
$$

In a nonequilibrium flow, the problem is to determine the energy level populations. The rotational and vibrational states in a molecule may be assumed to be populated according to distinct rotational and vibrational temperatures, respectively, if the nonequilibrium is not 
too severe. The electronic states, however, equilibrate more slowly so that a single electronic temperature cannot be defined. The electronic populations must then be calculated individually.

The emission coefficient in equilibrium is related to the absorption coefficient by Kirchhoff's law, which depends on the existence of an equilibrium temperature. This single temperature is not defined in nonequilibrium. Two approaches for finding the emission coefficient are possible. A quasi-equilibrium or excitation temperature appropriate to each radiative transition can be calculated, from which individual contributions to the emission can be obtained. This is the approach adopted in the NEQAIR code. ${ }^{5}$ Alternately, expressions for the emission coefficient can be found for nonequilibrium from detailed balancing. In principle, these two approaches should lead to the same result. However, the definition of an excitation temperature for use in the first approach is very sensitive to predicted nonequilibrium energy level populations that depend on rate data with significant uncertainties. An excitation temperature is defined by assuming that a Boltzmann or Saha equilibrium exists between the upper and lower level populations for a transition. For some nonequilibrium population distributions negative excitation temperatures can be computed. For bound-free transitions, for example, solving Saha's equation for $T$ gives:

$$
T_{e x}=\frac{I-E_{n}}{k \ln \left(N_{n} / N_{+} / N_{e} / f\left(T_{e}\right)\right)}
$$

If the nonequilibrium population of level $n$ is small the argument of the logarithm may be less than one (the function $f\left(T_{e}\right)$ in this equation is a combination of partition functions), resulting in a negative excitation temperature. Such negative temperatures have no physical meaning and therefore require that some justification be developed if they are to be used. The detailed balancing approach avoids this difficulty and is therefore employed in LORAN. The necessary expressions for the emission and absorption coefficients of the various radiative transitions are developed below. 


\section{Atomic Line Transitions}

The model selected for radiation absorption in atomic line transitions is that developed by Nicolet $^{6}$ for the RAD/EQUIL code. This model resolves a line by distributing about 15 spectral points starting from the line center as shown in Fig. 1. It has been modified as required for nonequilibrium. Following Nicolet's development, the absorption coefficient in a line is given by

$$
\kappa_{\nu}=\frac{\pi e^{2}}{m c} f_{l u} N_{l} b\left(\nu, N_{e}, T_{t}, T_{e} \ldots\right)
$$

The line shape $b$ is a normalized function that can be described by one of a number of theoretical models depending on the predominant broadening mechanism. Two such line shapes are considered in LORAN. The Lorentz shape describes lines in which Stark broadening by electron impact dominates, as is the case for heavy atomic species. In nonequilibrium this line shape is determined by the electron temperature. The mechanism of resonance broadening also yields Lorentz line shapes and may exceed Stark broadening when ionization is low. The second type of line shape is the Doppler profile, which describes line broadening due to thermal motion of the atoms, and is therefore determined by the heavy particle temperature. The details of these line shapes are given in Ref. 4.

The Lorentz and Doppler line shapes can be combined into a single curve, known as the Voigt line shape, which requires significantly more computation. Under the conditions of interest in this study, the two line widths have been found to be of different orders of magnitude. Therefore, it is felt that using the simpler line shape corresponding to the broadest line type should be adequate.

The radiative emission from a single atomic line is given by: ${ }^{7}$

$$
J^{e}=N_{u} h \nu_{u l} A_{u l}
$$

where $h \nu_{u l}$ is the energy difference in the transition. Detailed balancing relates $A_{u l}$ to the absorption oscillator strength $f_{l u}$ to give the emission coefficient as:

$$
j_{\nu}^{e}=\frac{2 \pi h e^{2}}{m c^{3}} \frac{g_{l}}{g_{u}} \nu_{u l}^{3} f_{l u} N_{u} b\left(\nu, N_{e}, T_{t}, T_{e} \ldots\right)
$$


The line shape $b$ is the same as that for absorption.

In the RAD/EQUIL code, Nicolet approximated the so-called high series lines by an integral. These lines have lower state quantum numbers of four or greater, and are numerous and closely spaced at energies below $3.2 \mathrm{eV}$. Park treats each of these lines individually in NEQAIR. In LORAN, the high series lines are represented by multiplet-averaged values in order to reduce the number of lines computed and therefore the run time. Data for these averaged lines were obtained from the NBS tables. ${ }^{8}$ This approximation should have minimal impact on the results, and yet allows the number of lines treated to be reduced by more than half.

\section{Atomic Bound-Free Transitions}

A model for bound-free transitions can be developed assuming a hydrogen-like atom (see for example Sec. 5.5 of Zel'dovich and Raizer ${ }^{7}$ ). The radiative cross sections developed there apply also to nonequilibrium since the configuration of an individual atom is unchanged:

$$
\sigma_{\nu n}=\frac{1}{\sqrt{3}} \frac{64 \pi^{4} m Z^{4} e^{10}}{3 \nu^{3} c h^{6} n^{5}}
$$

Once the cross section is known it is a simple matter to compute the spectral absorption coefficient for the bound-free process from Eq. 1, given the nonequilibrium populations of the electronic energy levels of the atom. At each frequency $\nu$, the summation will range from $n^{*}$, the photoionization threshold, to $n_{i}$, the ionization limit. $n^{*}$ is the lowest atomic energy level from which a photon with energy $h \nu$ can detach an electron. The grouped atomic

energy levels from Park's QSS method ${ }^{5}$ are used for nitrogen and oxygen atoms (twenty-two levels for atomic nitrogen, nineteen levels for atomic oxygen) to reduce book-keeping and complexity.

The hydrogen-like assumption may be in considerable error for atomic ground states. For many atoms, however, the cross section of the ground state is well known from experimental data. These data can be used for transitions involving the ground state instead of the predicted value from Eq. 6 . 
Bound-free emission results from the capture of a free electron into an ion. The cross section for this process is given by Zel'dovich and Raizer: ${ }^{7}$

$$
\sigma_{c n}=\frac{128 \pi^{4}}{3 \sqrt{3}} \frac{Z^{4} e^{10}}{m c^{3} h^{4} \mathrm{v}^{2} n^{3} \nu}
$$

where $\mathrm{v}$ is the initial speed of the captured electron. The number $n_{c}$ of such captures into the $n$th energy level for electrons with speeds between $\mathrm{v}$ and $\mathrm{v}+d \mathrm{v}$ per unit volume per unit time is

$$
n_{c}=N_{+} N_{e} f(\mathrm{v}) d \mathrm{v} \mathrm{v} \sigma_{c n}
$$

where $N_{+}$and $N_{e}$ are the ion and electron number densities, respectively. The electron speeds are distributed according to the distribution function $f(\mathrm{v})$, which is assumed to be a Maxwell distribution in equilibrium at the electron translational temperature $T_{e}$. The energy emitted, $h \nu_{u l}$, where the subscript $u$ now denotes the free state, is given by the sum of the initial kinetic energy of the electron and the net energy required to reionize it from the final energy level $l$.

$$
h \nu_{u l}=\frac{m \mathrm{v}^{2}}{2}+\left(I-E_{l}\right)
$$

This equation is solved for $\mathrm{v}^{2}$ and substituted to eliminate $\mathrm{v}$ in favor of the frequency $\nu$ in Eqs. 7 and 8.

Combining all these pieces, the emission coefficient for bound-free transitions is found from:

$$
j_{\nu}^{e} d \nu=\sum_{\text {final levels } 1} \frac{1}{4 \pi} h \nu_{u l} n_{c} d \nu
$$

The complete expression is

$$
\begin{array}{r}
j_{\nu}^{e}=\frac{128 \pi^{4}}{3 \sqrt{3}} \frac{Z^{4} e^{10}}{m^{2} c^{3} h^{2}} N_{+} N_{e}\left(\frac{m}{2 \pi k T_{e}}\right)^{3 / 2} \\
\sum_{n_{\min }}^{n_{i}} \frac{1}{n^{3}} \exp \left[-\left(\frac{h \nu-I+E_{l}}{k T_{e}}\right)\right]
\end{array}
$$

The lower limit on the summation corresponds to capture of a zero energy electron. $n_{\min }$ is determined for each frequency by setting v to zero in Eq. 9. The upper limit, $n_{i}$, corresponds to ionization of the atom. 


\section{Atomic Free-Free Transitions}

LORAN considers only the radiation produced by an electron slowed by the presence of an atomic ion. The free-free radiation produced by the proximity of a neutral atom or a molecule is considered negligible. A model for free-free transitions can be developed using semi-classical methods. ${ }^{7}$ The result can be rederived without equilibrium assumptions except for a Maxwell distribution of the energies of the free electrons at the electron translational temperature $T_{e}$. In this case, the only change in the result is that $T_{e}$ replaces $T$ :

$$
\kappa_{\nu}=\frac{4}{3}\left(\frac{2 \pi}{3 m k T_{e}}\right)^{1 / 2} \frac{Z^{2} e^{6}}{h c m \nu^{3}} N_{+} N_{e}
$$

Free-free transitions are a special case in nonequilibrium because they involve only free electrons. Since electrons are assumed to equilibrate rapidly to some temperature $T_{\epsilon}$, Kirchhoff's law and the development of the emission coefficient lead to the same result for emission:

$$
j_{\nu}^{e}=\frac{8}{3}\left(\frac{2 \pi}{3 m k T_{e}}\right)^{1 / 2} \frac{Z^{2} e^{6}}{m c^{3}} N_{+} N_{e} \exp \left(-h \nu / k T_{e}\right)
$$

\section{Molecular Transitions}

The treatment of molecular radiation requires consideration of vibrational and rotational transitions within the molecule, as well as electronic transitions; and combinations of the different types of transition. Each such transition contributes a discrete line to the radiation spectrum, whose line center frequency is determined by the energy differential of the transition. While a line-by-line calculation of molecular radiation is possible, it does not fit the objectives of the present study. As an alternative, therefore, the "smeared band" model of molecular radiation will be adopted here. In this model, the rotational line structure of the molecular radiation is smoothed to provide a continuous variation of the absorption co-

efficient. Because the rotational energy levels are so closely spaced, the lines resulting from rotational transitions are nearly overlapping except in very rarefied conditions. The error introduced by this approximation should therefore be acceptable under most conditions of interest. 
The basic development of the "smeared band" model can be found in a number of sources. The development outlined below is an enhancement of that found in Sec. 5.3 of Zel'dovich and Raizer. ${ }^{7}$ It incorporates higher order expressions for the molecular energy levels from Herzberg, ${ }^{9}$ while eliminating equilibrium assumptions. In what follows ZR will refer to the text by Zel'dovich and Raizer. Herzberg will refer to the just-quoted reference. The complete details of the development are given in Ref.4.

The energy differentials for the molecular transitions will be established first. The complete molecular energy level is $E_{t o t}=E_{e}+E_{v}+E_{r}$, where the electronic energy levels are given by $E_{n}$, the energy of a particular electronic level (measured from the ground state); the vibrational energy levels, $E_{v}$, are obtained from the fourth order expression given by Herzberg that includes a correction for the anharmonicity of the vibrational energy potential; and the energy levels for the rotational transitions, $E_{r}$, including a correction for non-rigid oscillators, are also given in Herzberg.

Designating the upper level by the superscript $u$ and the lower level by $l$, the wave number (inverse wavelength) of a particular transition is

$$
\frac{1}{\lambda}=\frac{E_{t o t}^{u}-E_{t o t}^{l}}{h c}
$$

For convenience in manipulating these expressions one special wave number is defined. This is the wave number with zero rotational quantum number and arbitrary vibrational quantum numbers, $1 / \lambda_{v^{u} v^{l}}$.

The formidable expression for the wave number of an individual transition is simplified by the selection rules for rotation. These rules are discussed in various sources including ZR and Herzberg, and result in the requirement that $\Delta J=J^{u}-J^{l}=0,+1$, or -1 . In addition, the $0-0$ rotational transition is forbidden and in the case of transitions between certain molecular states denoted by $\Sigma$ all $\Delta J=0$ transitions are forbidden. A detailed discussion of these selection rules and of the designation of molecular states is outside the scope of the present work. Nevertheless, these rules allow the development of three specific wave numbers for the $\Delta J=-1,0,1$ transitions, which are denoted $\mathrm{P}, \mathrm{Q}$, and $\mathrm{R}$, respectively. If the $D_{e}$ 
terms in the rotational energy level expression of Herzberg are neglected in comparison with the $B_{v}$ terms, and if $J^{l} \gg 1$, which is the case for most lines, is assumed, the three wave numbers for $\mathrm{P}, \mathrm{Q}$, and $\mathrm{R}$ transitions collapse into the single form:

$$
\frac{1}{\lambda}=\frac{\nu}{c} \simeq \frac{1}{\lambda_{v^{u} v^{l}}}+J^{l^{2}}\left(B_{v}^{u}-B_{v}^{l}\right)
$$

This equation defines the spectral location of each rotational line in each vibrational band for each pair of electronic states. Retaining terms linear in $J$ would increase the accuracy of this expression without requiring much additional work, but given the level of uncertainty in the radiation properties Eq. 15 is felt to be sufficiently accurate.

Once the molecular band spectrum is thus determined, it is necessary to evaluate the probability of a transition between levels in order to obtain an expression for the absorption coefficient. This probability is obtained from a quantum mechanical analysis. The resulting expression for the absorption coefficient is given for instance in ZR:

$$
\begin{array}{r}
\kappa_{\nu_{A v^{l} J} l_{B v^{u} J^{u}}}=\frac{g_{B v^{u} J^{u}}}{g_{A v^{l} J^{l}}} \frac{8 \pi^{3}}{3 h c} N_{A v^{l} J^{l}} D_{e l B A}^{2} \\
\nu_{A v^{l} J^{l}, B v^{u} J^{u}} q_{v^{u} v^{l}} p_{J^{u} J^{l}} F(\nu)
\end{array}
$$

where $A$ and $B$ denote the lower and upper electronic states. This expression must be evaluated for each $A, B, v^{u}, v^{l}, J^{u}, J^{l}$ in order to obtain the complete absorption spectrum of a molecular species. The "smeared band" model averages out the rotational line structure. This is done by expanding the degeneracy $g$ as $g=g_{e} g_{v} g_{r} \approx g_{e}(2 J+1)$, summing Eq. 16 over $J^{u}$, and introducing an average frequency, $\bar{\nu}_{B v^{u}, A v^{l}}$, for the rotational lines in a vibrational band. The approximation $J \gg 1$ is again used here, even for transitions with small $J$. While the error introduced in a single transition with a low rotational quantum number can be as much as 300 percent only a few transitions have low $J$ so the effect on the overall absorption coefficient is small. The error incurred by introducing the average frequency $\bar{\nu}_{B v^{u}, A v^{l}}$ for all rotational lines in the $v^{l}-v^{u}$ band is minimal, on the order of one to seven percent.

Proceeding towards the complete absorption coefficient, Eq. 16 is then summed over the final vibrational level $v^{u}$. Here the frequency remains inside the summation and is not 
averaged, as the variation involved can be large. The mean value theorem is then used to define an average absorption coefficient for a line, by integrating over one line width $\Delta \nu$ :

$$
\begin{array}{r}
\int_{l i n e} \kappa_{\nu_{A v^{l} J^{l}, B}} d \nu=\bar{\kappa}_{\nu_{A v^{l} J^{l}, B}} \Delta \nu \\
=\frac{8 \pi^{3}}{3 h c} \frac{g_{B}}{g_{A}} N_{A v^{l} J^{l}} D_{e l B A}^{2} \sum_{v^{u}}^{\infty} q_{v^{u} v^{l}} \bar{\nu}_{B v^{u}, A v^{l}}
\end{array}
$$

Equation 17 is solved for $\bar{\kappa}_{\nu}$ and summed over the initial rotational and vibrational levels, where the sum is zero for any transition that does not absorb radiation in the frequency interval under consideration. An expression for $\Delta \nu$ can be obtained from Eq. 15 . The final result for the "smeared band" molecular absorption coefficient is

$$
\begin{array}{r}
\bar{\kappa}_{\nu_{A B}}=\frac{8 \pi^{3}}{3 h c} \frac{g_{B}}{g_{A}} D_{e l B A}^{2} \sum_{v^{l}}^{\infty} \sum_{v^{u}}^{\infty} q_{v^{u} v^{l}} \bar{\nu}_{B v^{u}, A v^{l}} \\
\sum_{J^{l}}^{\infty} N_{A} \frac{\exp \left(-E_{v^{l}} / k T_{v}\right)}{Q_{v}\left(T_{v}\right)} \frac{1}{c\left|B_{v^{u}}-B_{v^{l}}\right| Q_{r}\left(T_{r}\right)} \\
\exp \left(-\frac{h c}{k T_{r}}\left\{\left[B_{e}^{l}-\alpha_{e}^{l}\left(v^{l}+\frac{1}{2}\right)\right]\left(\frac{1}{\lambda}-\frac{1}{\lambda_{v^{u} v^{l}}}\right)\right.\right. \\
\left.\left.\frac{1}{B_{v^{u}}-B_{v^{l}}}-\frac{4 B_{e}^{l^{3}}}{\omega_{e}^{l^{2}}}\left(\frac{1}{\lambda}-\frac{1}{\lambda_{v^{u} v^{l}}}\right)^{2} \frac{1}{\left(B_{v^{u}}-B_{v^{l}}\right)^{2}}\right\}\right)
\end{array}
$$

This expression is to be evaluated for each pair of electronic states $A$ and $B$. As currently implemented, vibrational levels for all species are assumed to be populated according to a single $T_{v}$, and rotational levels to a single $T_{r}$. If reliable species $T_{v}$ and $T_{r}$ later become available from CFD codes, they can easily be used in the radiation calculation.

The averaged frequency, $\bar{\nu}_{B v^{u}, A v^{l}}$, must be evaluated separately for non- $\Sigma$ and $\Sigma$ transitions according to the rotational selection rules discussed above. It is obtained by summing over all allowable transitions, then dividing by the number of such transitions. The results are:

$$
\begin{array}{r}
\bar{\nu}_{B v^{u}, A v^{l}}(\text { non- } \Sigma)=c\left[\frac{1}{\lambda_{v^{u} v^{l}}}+\frac{2 B_{v}^{u}\left(J_{\text {max }}^{l}+1\right)}{3 J_{\text {max }}^{l}+1}\right. \\
\left.+\frac{3\left(B_{v}^{u}-B_{v}^{l}\right)}{3 J_{\max }^{l}+1} J_{\text {max }}^{l}\left(J_{\text {max }}^{l}+1\right)\left(J_{\text {max }}^{l}+2\right)\right]
\end{array}
$$




$$
\begin{aligned}
& \bar{\nu}_{B v^{u}, A v^{l}}(\Sigma)=c\left[\frac{1}{\lambda_{v^{u} v^{l}}}+\frac{2 B_{v}^{u}\left(J_{\max }^{l}+1\right)}{2 J_{\text {max }}^{l}+1}\right. \\
& \left.+\frac{2\left(B_{v}^{u}-B_{v}^{l}\right)}{2 J_{\max }^{l}+1} J_{\text {max }}^{l}\left(J_{\max }^{l}+1\right) \frac{\left(J_{\max }^{l}+2\right)}{3}\right]
\end{aligned}
$$

where $J_{\max }$ is the maximum rotational quantum number before the molecule dissociates. The $D_{e}$ terms in the rotational energy have again been neglected.

The term inside the final summation in Eq. 18 is the population of the lower energy level involved in the transition. This has been expanded from

$$
N_{A v^{l} J^{l}}=N_{A} \frac{\exp \left(-E_{v^{l}} / k T_{v}\right)}{Q_{v}\left(T_{v}\right)} \frac{g_{J^{l}} \exp \left(-E_{J^{l}} / k T_{r}\right)}{Q_{r}\left(T_{r}\right)}
$$

using an approximate expression for $J$ in terms of wave number from Eq. 15. This imposes the requirement for $B_{v^{u}}>B_{v^{l}}$ that $\lambda_{v^{u} v^{l}}>\lambda$, and for $B_{v^{u}}<B_{v^{l}}$ that $\lambda_{v^{u} v^{l}}<\lambda$. These requirements are the mathematical statement of the fact that each vibrational band absorbs in only a portion of the spectrum. In evaluating the absorption coefficient for a particular band, the wavelength or frequency under consideration must be checked against these conditions to avoid adding superfluous contributions. The population of the electronic energy level $N_{A}$ must be obtained from some nonequilibrium excitation calculation. In the present work, Park's QSS method, ${ }^{10}$ discussed briefly below, has been used.

A "smeared band" expression can be developed for the molecular emission coefficient by a process parallel to that for absorption. The total emission in a rotational line, $J_{u l}^{e}$, is obtained from the Einstein coefficient for spontaneous emission according to Eq. 4 . The emission intensity at a particular frequency is obtained by dividing by the solid angle and introducing a line shape function, $F(\nu)$.

$$
j_{\nu_{u l}}^{e}=\frac{N_{u} A_{u l} h \nu_{u l}}{4 \pi} F(\nu)
$$

Substituting the Einstein coefficient, this becomes

$$
j_{\nu_{u l}}^{e}=N_{u} \frac{16 \pi^{3}}{3 c^{2}} \nu_{u l}^{4} D_{e l B A}^{2} q_{v^{u} v^{l}} p_{J^{u} J^{l}} F(\nu)
$$

To obtain the "smeared band" result, this expression must first be summed over $J^{l}$ and the average frequency $\bar{\nu}_{B v^{u}, A v^{l}}$ introduced. Proceeding in parallel to the development for 
absorption, the sum over $v^{l}$ is then evaluated. The average emission coefficient for a single rotational line is now obtained using the mean value theorem. This mean value is then summed over the final rotational and vibrational levels, $J^{u}$ and $v^{u}$. The "smeared band" result for emission is then:

$$
\begin{array}{r}
\bar{j}_{\nu_{A B}}^{e}=\frac{16 \pi^{3}}{3 c^{2}} D_{e l B A}^{2} \sum_{v^{u}}^{\infty} \sum_{v^{l}}^{\infty} q_{v^{u} v^{l}} \bar{\nu}_{B v^{u}, A v^{l}}^{4} \\
\sum_{J^{u}}^{\infty} N_{B} \frac{\exp \left(-E_{v^{u}} / k T_{v}\right)}{Q_{v}\left(T_{v}\right)} \frac{1}{c\left|B_{v^{u}}-B_{v^{l}}\right| Q_{r}\left(T_{r}\right)} \\
\exp \left(-\frac{h c}{k T_{r}}\left\{\left[B_{e}^{u}-\alpha_{e}^{u}\left(v^{u}+\frac{1}{2}\right)\right]\left(\frac{1}{\lambda}-\frac{1}{\lambda_{v^{u} v^{l}}}\right)\right.\right. \\
\left.\left.\frac{1}{B_{v^{u}}-B_{v^{l}}}-\frac{4 B_{e}^{u^{3}}}{\omega_{e}^{u^{2}}}\left(\frac{1}{\lambda}-\frac{1}{\lambda_{v^{u} v^{l}}}\right)^{2} \frac{1}{\left(B_{v^{u}}-B_{v^{l}}\right)^{2}}\right\}\right)
\end{array}
$$

The average frequency, $\bar{\nu}_{B v^{u}, A v^{l}}$, is the same as that for absorption, and is given by Eqs. 19 and 20 for non- $\Sigma$ and $\Sigma$ transitions, respectively. The final term in the emission coefficient, the nonequilibrium upper level population, is found in the same manner as the lower level population for absorption.

\section{Induced Emission}

The above expressions for the emission coefficient do not include all the radiation emitted by the medium. Stimulated or induced emission also occurs due to the presence of photons. This emission is proportional to the radiative intensity, $I_{\nu}$, and is therefore commonly included as a correction to the absorption coefficient. The induced emission is given by:

$$
j_{\nu}^{\prime}=j_{\nu}^{e} \frac{c^{2}}{2 h \nu^{3}} I_{\nu}
$$

The nonequilibrium corrected absorption coefficient is therefore:

$$
\kappa_{\nu}^{\prime}=\kappa_{\nu}-j_{\nu}^{e} \frac{c^{2}}{2 h \nu^{3}}
$$

The corrected absorption coefficient may be negative in nonequilibrium. Negative values of $\kappa_{\nu}^{\prime}$ occur when the nonequilibrium populations are such that the second term on the righthand-side of Eq. 26 is larger than the first term. Physically, this means that the upper energy 
level of a transition or transitions is overpopulated relative to the lower energy level. Such a population inversion can occur in a nonequilibrium boundary layer, for instance, when the higher energy levels are still populated according to the temperature and chemistry of the inviscid shock layer. Because of the $\nu^{-3}$ term appearing in Eq. 26, negative values of $\kappa_{\nu}^{\prime}$ tend to occur at the low energy end of the spectrum. The occurrence of these negatives must be carefully considered when developing any radiative transport algorithm for nonequilibrium media.

\section{Nonequilibrium Excitation}

The nonequilibrium excitation portion of the present method was taken from Park's NEQAIR program. It consists of a quasi-steady-state (QSS) solution to the set of excitation rate equations, including collisional and radiative transitions. The QSS method assumes that the rate of change of a level's population is small compared with the rate of transitions into and out of that level. ${ }^{10}$ This method is designed for use in situations where the amount of nonequilibrium is "not too large". Its most serious weakness in the present application is that it does not account for the history of the flow in areas of high gradients (boundary layer and shock).

The excitation calculation depends on excitation rate data for the various energy levels. This type of data is difficult to obtain and therefore has a significant level of uncertainty. Park $^{11}$ cites this uncertainty as a factor of two, and asserts that the impact on the excited state populations is negligible. Further verification of this accuracy would be desirable in the future.

\section{Optimization}

\section{Radiation Spectrum}

The spectral location of all radiating transitions is determined from the set of energy levels in the atoms and molecules appearing in the gas. This information can be used to select a minimum set of spectral points for the radiation calculation. 
Consider the bound-free continuum. The absorption coefficient for this mechanism is a smooth function of frequency with discontinuities corresponding to the activation of additional energy levels. To resolve this spectrum, spectral points must be placed within a very small interval on either side of each jump, as shown in Fig. 2. However, this interval is much smaller than what is needed for the smooth portions of the curve. RAD/EQUIL took advantage of this fact by allowing the user to input a few spectral points to capture the jumps. In LORAN, this procedure is automated and incorporated in the radiation calculation. For the particular set of atomic species considered in a computation, a tailored atomic spectrum is generated to resolve the active discontinuities, as Fig. 2 illustrates. This simplifies the inputs and eliminates the chances of omitting a level. It also allows the atomic continuum calculation to be performed on the minimum resolving array of spectral points.

For the atomic line spectrum, each line is represented at each grid point in the flowfield by a small number $(n p p l)$ of points distributed according to the larger of the Doppler and Lorentz line widths calculated from the local conditions. (In RAD/EQUIL, the point distribution logic considered only the maximum Stark line width of each line.) As seen in Fig. 1, this point distribution is sufficient to capture the shape of the line and most of the energy it contains. The disparate frequency spectra obtained throughout the flowfield are then reconciled to generate a single atomic line spectrum. This process results in the selection of a set of spectral points capturing the energy in a line at its broadest, but involving detailed spectral information at each grid point. It allows satisfactory resolution of the atomic lines without requiring an excessive amount of storage or computation.

The molecular radiation calculation is traditionally the most expensive, consisting as it does of the combined effects of $10^{5}$ to $10^{6}$ rotational lines. The "smeared band" model averages over this rotational structure and results in an absorption coefficient that varies smoothly over vibrational bands with discontinuities at the various band heads. The resulting structure is similar to the bound-free spectrum, though it is much more complicated because the vibrational bands are far more numerous and often overlap each other. In order to resolve 
this spectrum, a similar approach has been taken. For each active species, the vibrational band heads that occur in the spectral range under study are computed from Eq. 15 with $J=0$. An initial spectrum is generated by resolving the band head and spacing nv points in the interval between the zero rotational level $(J=0)$ and the maximum rotational level $\left(J=J_{\max }\right)$. An example for a major vibrational band of $N_{2}^{+}$is given by the curve labeled "initial spectrum" in Fig. 3. Note that the clustering of points along this curve results from additional weaker bands that underlie this major band.

Since the absorption coefficient decreases rapidly for a given vibrational band (note the $\log$ scale in the figure), this initial spectrum can be reduced. This is done by discarding points that describe a vibrational band away from its band head, if they are within a given interval, crit, of another point. This procedure can reduce the spectral array by nearly an order of magnitude. Applying this process to the spectrum in Fig. 3 results in the optimized spectrum shown. Applying it to the entire set of vibrational bands, a spectrum can be generated that resolves the molecular transitions using less than 2000 points and that is optimized for whatever species and spectral range have been requested. This calculation is computationally expensive, since it sifts through a large number of possible spectral points to produce the optimized molecular spectrum, and it is therefore only exercised once per run. The resulting spectrum is stored for all further computations. Appropriate selection of the parameters $n v$ and crit controls the amount of detail included in the molecular spectrum and its size.

\section{Radiation Calculation}

Parametric studies were conducted to determine the spectral resolution required for acceptable accuracy in the predicted radiation. Variables whose effect was studied include $n a$, the number of points allowed in the atomic continuum spectrum; nppl, the number of points resolving an atomic line; and the variables that control the generation of the spectrum for molecular band radiation ( $n v$ and $c r i t$ ). The three FIRE II flight conditions of Ref. 3 
were studied to obtain results over a wide range of nonequilibrium conditions. Only the results for at $1637.5 \mathrm{sec}$, which showed the most variation, are presented here.

Table 1 summarizes the findings of this optimization process. The baseline (first line of the table) against which the flux calculation is judged is a detailed spectrum case with $n a=1500, n p p l=15, n v=10$, and crit $=0.00825 \mathrm{eV}$. It is assumed that these values provide a near-exact heat flux. The run times are judged against a case with $n a=100$ and all other values unchanged (second line of the table), since this CPU time is more in line with the objective of a rapid calculation. Column 1 specifies the new value of the parameter that was changed from the baseline. The designations $\mathrm{C} 1$ and $\mathrm{C} 2$ refer to combinations of changes as follows: $\mathrm{C} 1$ has $n a=75, n p p l=11, n v=8$, and crit $=0.0165 \mathrm{eV}$; $\mathrm{C} 2$ is the same as $\mathrm{C} 1$ except $n a=100$.

Table 1: Computational Optimization

\begin{tabular}{ccccc}
\hline \hline Case & Heat & CPU for & CPU for & CPU \\
& Flux & Radiation & Transport & Fraction \\
\hline Baseline & 292.9 & 10.061 & 2.913 & 0.73 \\
$n a=100$ & $+1.5 \%$ & 5.754 & 1.617 & 0.60 \\
$n p p l=11$ & $+1.9 \%$ & $-12.1 \%$ & $-28.9 \%$ & 0.56 \\
$n a=75$ & $+6.1 \%$ & $-1.2 \%$ & $-1.1 \%$ & 0.60 \\
$n v=8$ & $+1.2 \%$ & $-0.1 \%$ & $+0.1 \%$ & 0.60 \\
$c r i t * 2$ & $+1.6 \%$ & $-0.7 \%$ & $+0.2 \%$ & 0.60 \\
$\mathrm{C} 1$ & $+6.3 \%$ & $-14.1 \%$ & $-30.2 \%$ & 0.55 \\
$\mathrm{C} 2$ & $+1.7 \%$ & $-24.5 \%$ & $-28.8 \%$ & 0.53 \\
\hline \hline
\end{tabular}

The prediction of the radiative flux is affected by less than 5 percent for each set of parameters, except for the two occurrences shown in Table 1 at 1637.5 sec. These are both attributable to setting $n a=75$. The potential savings in computational time are considerable. 
Based on these results, it is determined that the parameter set denoted $\mathrm{C} 2(\mathrm{na}=100$, $n p p l=11, n v=8$, crit $=0.0165 \mathrm{eV}$ ) is sufficient for use in the radiation model. Note that this parameter set results in a higher, thus more conservative, prediction of radiation. When accuracy is more important than saving CPU time, it is easy to change these parameters to improve the result.

\section{Excitation Calculation}

The last column in Table 1 shows the fraction of CPU time used by the radiation calculation. This fraction is about 0.55 at 1634 sec, and 0.36 at 1631 sec. The remainder of the CPU time is required to obtain the nonequilibrium excited state populations, and is a significant part of the total. The QSS algorithm used to perform this calculation was obtained from NEQAIR and used as a "black box". While important savings may be possible in this algorithm, no attempt has been made to achieve them. It is clear that this is a limiting factor in the efficiency of the radiation prediction.

\section{Radiation Subgrid}

Calculating radiative properties at every point on a flowfield grid is often wasteful. Absorption and emission only change significantly in regions where the temperatures or species concentrations have large gradients. Bolz ${ }^{12}$ developed an algorithm to automatically select a subset of grid points for the radiation calculation. He defines a weighting function that can be adapted to the nonequilibrium situation as

$$
\begin{array}{r}
Z_{k}=k+\sum_{i=1}^{k}\left[W_{C} \frac{\bar{C}_{i}^{\prime}}{\bar{C}_{i, \max }^{\prime}}+W_{T} \frac{\left|T^{\prime}\right|_{i}}{\left|T^{\prime}\right|_{i, \text { max }}}\right. \\
\left.+W_{T_{v}} \frac{\left|T_{v}^{\prime}\right|_{i}}{\left|T_{v}^{\prime}\right|_{i, \text { max }}}+W_{T_{e}} \frac{\left|T_{e}^{\prime}\right|_{i}}{\left|T_{e}^{\prime}\right|_{i, \text { max }}}+W_{q_{R}} \frac{\left|q_{R}^{\prime}\right|_{i}}{\left|q_{R}^{\prime}\right|_{i, \text { max }}}\right]
\end{array}
$$

The ' in these equations denotes the partial derivative with respect to $\eta$, the normal to the wall. The average species concentration derivative is

$$
\bar{C}_{i}^{\prime}=\sum_{j=1}^{N}\left|C_{i, j}^{\prime}\right|
$$


for $N$ chemical species. Though weighting factors are included for each term in $Z_{k}$ there is no obvious reason to emphasize the gradient in one variable more than that of another. The $W_{i}$ are therefore all set equal to one.

The subgrid algorithm has been assessed using the nonequilibrium test cases. The effects are summarized in Table 2. The subgrid results (columns 3 and 4) were obtained using the optimized $\mathrm{C} 2$ set of parameters discussed above, while the detailed spectrum result (column 2) used the Baseline parameter set to establish a near-exact reference heat flux. The CPU time required for a given case is a nearly linear function of the number of grid points, so the time savings can easily be assessed.

Table 2: Effect of Radiation Subgrid Algorithm

\begin{tabular}{cccc}
\hline \hline FIRE II & Detail spectrum & 20 & 15 \\
Point & All grid points & grid points & grid points \\
\hline $1631 \mathrm{sec}$ & 0.508 & $-1.4 \%$ & $+6.4 \%$ \\
$1634 \mathrm{sec}$ & 22.50 & $+10.7 \%$ & $+14.2 \%$ \\
$1637.5 \mathrm{sec}$ & 292.9 & $+3.9 \%$ & $+2.9 \%$ \\
\hline \hline
\end{tabular}

For these cases using twenty grid points (out of 64 along normal grid lines in the flowfield) selected with the modified Bolz algorithm provides radiative heat flux predictions within about 10 percent of the reference result, even including the spectral approximations introduced by the use of the $\mathrm{C} 2$ data set. A subgrid of this size is therefore considered adequate, confirming Bolz' conclusions. Again, when accuracy is more important than computational efficiency, the size of the subgrid can easily be increased.

\section{Verification}

Gas radiation is a complex phenomenon, so that true verification of a radiation model requires checking it against a large number of flight and ground-based experiments covering a 
wide range of conditions. Only a few such measurements are currently available. Representative results for these cases are shown here as an initial test of the method. All nonequilibrium flowfields used in the verification were generated by the LAURA code of Gnoffo, ${ }^{1}$ in order to eliminate differences caused by flowfield modeling.

\section{AVCO Shock Tube Data}

One of the classic radiation measurements is that in the AVCO shock tube. ${ }^{13}$ Using the gas conditions suggested by Park, ${ }^{14}$ a predicted spectrum for the emission intensity has been obtained for the peak radiation point, and is shown in Fig. 4 by the solid line. This figure also reproduces the NEQAIR prediction from the just-cited reference and the original AVCO data. The agreement between the measured data and the present result is no worse than that shown by NEQAIR and may actually be a little better. (The discrepancy at the shortest wavelengths is unexplained. It is attributed to contamination of the measurement since air radiates weakly in that spectral region.) The AVCO data exhibit significant scatter, so only a qualitative assessment can be made. The atomic lines appearing in Fig. 4 are multipletaveraged, and so should exhibit only gross agreement with the NEQAIR prediction, as is observed.

\section{FIRE Project}

The FIRE flight project of the mid-1960's consisted of two flights simulating reentry of an Apollo type vehicle. A discussion of the flights is given in a previous paper, ${ }^{3}$ which also presented predicted spectra from LORAN and compared them to the measured flight data. Two points in the FIRE II trajectory have been examined for the present work: 1634 and $1637.5 \mathrm{sec}$. Flowfield solutions were obtained with LAURA for the appropriate flight conditions. The predicted stagnation line radiative emission profiles, for the spectral range in which the radiometer windows transmit (0.31 to $5.4 \mathrm{eV})$, are shown in Fig. 5. Calculations from LORAN and NEQAIR are compared, broken out into the various radiating mechanisms. The NEQAIR result shown here was computed using a version of the NEQAIR code that 
was obtained from Chul Park in 1988. It has been slightly modified to correct some minor errors this version contained, and to allow emission calculations within a limited spectral range. The agreement between the two predictions is excellent (in fact the curves lay on top of each other through much of the layer) except for a discrepancy in the atomic line radiation near the wall. The predicted wall radiative heat flux differs by only 3 percent between the two codes.

Figure 6 shows the emission profiles for the 1637.5 sec case. The overall agreement between the two predictions is quite good, although there are some differences. The radiative flux reaching the wall varies by about 6 percent between these two predictions.

Radiation predictions for these cases have also been made by Carlson and Gally ${ }^{15}$ for a nitrogen freestream. Figure 7 can be qualitatively compared with Figure 5 of their paper. It shows the approximate spectral variation of the wall radiative flux over the entire spectrum including the ultraviolet. Though the predicted magnitudes are lower in Ref. 15 because only nitrogen is considered, the variations are nonetheless qualitatively very similar. The details of the spectra cannot be compared because of the line group presentation used in the reference. It should also be noted that the chemical kinetics and other models used by Carlson and Gally are different from those in the LAURA code. As demonstrated in a previous paper, ${ }^{3}$ these models can have a large impact on the magnitude and spectral distribution of the predicted radiative heating.

\section{Flowfield Coupling Strategy}

Including the gas radiation effects in a thermochemical nonequilibrium flowfield calculation involves coupling the fluid dynamic computation (from LAURA, ${ }^{1,2}$ for instance) with the radiation calculation from LORAN. The coupling occurs because of the radiative flux term $\nabla \cdot \vec{q}_{R}$ that appears in the total energy and vibrational-electronic energy equations. This term is computed by LORAN for each point in the flowfield, either directly or by interpolation of results on a radiation subgrid. Coupling can be accomplished by alternatively 
computing the flowfield with LAURA and the radiative flux term with LORAN. Because the computation of the radiation requires up to three orders of magnitude more computational time than one iteration of the fluid dynamics computation, however, it is advantageous to utilize a multitasking strategy. Multiple tasks are assigned to the radiation calculation while a single task computes the nonequilibrium flowfield. The computational domain is partitioned into three to seven subdomains (depending on the number of processors available), each assigned to a processor for calculation of the radiation field. When run asynchronously with the single flowfield task, the multiple radiation tasks allow the flowfield equations access to the latest radiative flux terms in shared memory (multiple LAURA iterations still occur during a single radiation update). This asynchronous strategy is an efficient use of a multiple processor machine since no processor remains idle waiting for another task. Preliminary computational experiments indicate no instabilities in this approach. The effectiveness of asynchronous multitasking schemes using LAURA is further discussed in Ref.16.

As a practical matter, it is more efficient to first obtain a converged flowfield solution using LAURA, then "turn on" radiation and converge the coupled flowfield. If the radiation is only a small perturbation to the flowfield this second convergence may require only a few additional LAURA iterations. If there are strong radiation effects then converging the coupled flowfield will require many more iterations. To investigate the practicality of this approach an initial radiation solution was obtained for a $60^{\circ}$ sphere cone with a $1.08 \mathrm{~m}$ nose radius at a velocity of $12 \mathrm{~km} / \mathrm{sec}$ and an altitude of $80 \mathrm{~km}$. The predicted convective and radiative heating distributions are shown in Fig. 8. (The variation of $q_{R}$ does not contradict the very limited flight data obtained from Project FIRE.) With the optimized LORAN code, this solution requires $176 \mathrm{CPU}$ seconds on an IRIS 4D/320 workstation. Obtaining converged coupled solutions is therefore computationally practical. Like all the results presented in this paper, these were obtained using the tangent slab approximation to compute radiative transport along each normal grid line. Work is well under way on a method to remove the tangent slab assumption, as reported elsewhere. ${ }^{4,17}$ Additional results 
showing the effect of flowfield models on the prediction of radiative heating for the above case are given by Mitcheltree. ${ }^{18}$

\section{Conclusions}

The development of the LORAN code, a nonequilibrium radiation method for use in coupled flowfield solutions, has been reported. Issues which arose during its development have been discussed. Strategies used to resolve these issues have been presented. Representative results comparing the method to available ground and flight data indicate that LORAN predicts radiation with accuracy similar to that of Park's NEQAIR code ${ }^{5}$.

Early results including flowfield coupling demonstrate the feasibility of using LORAN coupled to a detailed nonequilibrium CFD code such as LAURA. Additional work is needed to make it a more efficient tool for computing nonequilibrium flowfields with coupled radiation, especially when coupling effects are significant and many iterations are required to converge the coupled solution. Work is continuing to further reduce the time required to compute radiation by applying vectorization and other efficient strategies.

\section{Acknowledgments}

This work was performed as part of a doctoral program under the direction of Dr. Fred R. DeJarnette and Dr. Hassan A. Hassan of North Carolina State University. The assistance of Dr. Robert A. Mitcheltree, of the NASA Langley Research Center, in coupling the LORAN and LAURA codes is gratefully acknowledged.

\section{References}

${ }^{1}$ Gnoffo, P. A., "Upwind-Biased Point-Implicit Relaxation Strategies for Viscous Hypersonic Flows," AIAA Paper 89-1972, June, 1989.

${ }^{2}$ Gnoffo, P. A., Gupta, R. N., and Shinn, J. L., "Conservation Equations and Physical Models for Hypersonic Air Flows in Thermal and Chemical Nonequilibrium", NASA TP 2867, February, 1989. 
${ }^{3}$ Hartung, L. C., Mitcheltree, R. A., and Gnoffo, P. A., “ Stagnation Point Nonequilibrium Radiative Heating and the Influence of Energy Exchange Models", AIAA Paper 91-0571, January, 1991.

${ }^{4}$ Hartung, L. C., “Nonequilibrium Radiative Heating Prediction Method for Aeroassist Flowfields with Coupling to Flowfield Solvers", PhD Thesis, North Carolina State University, 1991.

${ }^{5}$ Park, C., “Nonequilibrium Air Radiation (NEQAIR) Program: User's Manual", NASA TM-86707, July, 1985.

${ }^{6}$ Nicolet, W. E., “Advanced Methods for Calculating Radiation Transport in AblationProduct Contaminated Boundary Layers", NASA CR-1656, September, 1970.

'Zel'dovich, Ya. B., and Raizer, Yu. P., "Physics of Shock Waves and High-Temperature Hydrodynamic Phenomena", Vol. 1, Academic Press, New York, 1966.

${ }^{8}$ Wiese, W. L., Smith, M. W., and Glennon, B. M., "Atomic Transition Probabilities; Volume I: Hydrogen Through Neon", National Bureau of Standards, NSRDS-NBS 4, May, 1966.

${ }^{9}$ Herzberg, G., “Molecular Spectra and Molecular Structure. I. Spectra of Diatomic Molecules", D. Van Nostrand Co., Inc., Princeton, New Jersey, 1950.

${ }^{10}$ Park, C., "Nonequilibrium Hypersonic Aerothermodynamics, Wiley, New York, 1990.

${ }^{11}$ Park, C., "Calculation of Nonequilibrium Radiation in AOTV Flight Regimes", AIAA Paper 84-0306, January, 1984.

${ }^{12}$ Bolz, C. W., Jr., “An Algorithm for Selecting a Radiation Transport Subgrid for Ablation and Radiation Coupled Hypersonic Viscous Shock Layer Problems", NASA CR144957, March, 1976.

${ }^{13}$ Allen, R. A., Rose, P. H., and Camm, J. C., "Nonequilibrium and Equilibrium Radiation at Super-Satellite Re-entry Velocities", Avco-Everett Research Laboratory, Research Report 156, BSD-TDR-62-349, September, 1962.

${ }^{14}$ Park, C. “Assessment of Two-Temperature Kinetic Model for Ionizing Air", Journal 
of Thermophysics and Heat Transfer, Vol. 3, No. 3, July, 1989, pp. 233-244.

${ }^{15}$ Carlson, L. A., and Gally, T. A., "Nonequilibrium Chemical and Radiation Coupling

Phenomena in AOTV Flowfields", AIAA Paper 91-0569, January, 1991.

${ }^{16}$ Gnoffo, P. A., "Asynchronous, Macrotasked Relaxation Strategies For the Solution of Viscous Hypersonic Flows", AIAA Paper 91-1579, June, 1991.

${ }^{17}$ Hartung, L. C., and Hassan, H. A., "Radiation Transport Around Axisymmetric Blunt Body Vehicles Using a Modified Differential Approximation", AIA A Paper 92-0119, January, 1991.

${ }^{18}$ Mitcheltree, R. A., "A Parametric Study of Dissociation and Ionization Models at 12 km/sec", AIAA Paper 91-1368, June, 1991. 


\section{Figure Captions}

1. Distribution of Spectral Points for an Atomic Line

2. Example of Spectrum Optimization for Bound-Free Continuum

3. Example of Spectrum Optimization for a Vibrational Band

4. Calculated Spectrum for the Peak Radiation Point of the AVCO Experiment

5. Emission Profiles for FIRE II at $1634 \mathrm{sec}$ with Comparison to NEQAIR

6. Emission Profiles for FIRE II at $1637.5 \mathrm{sec}$ with Comparison to NEQAIR

7. Spectral Variation of Stagnation Point Radiative Heat Transfer for FIRE II

8. Variation of Convective and Radiative Heat Transfer on a $60^{\circ}$ Sphere Cone with $R_{N}=1.08 \mathrm{~m}, \mathrm{~V}=12 \mathrm{~km} / \mathrm{sec}$ and $\mathrm{H}=80 \mathrm{~km}$ 


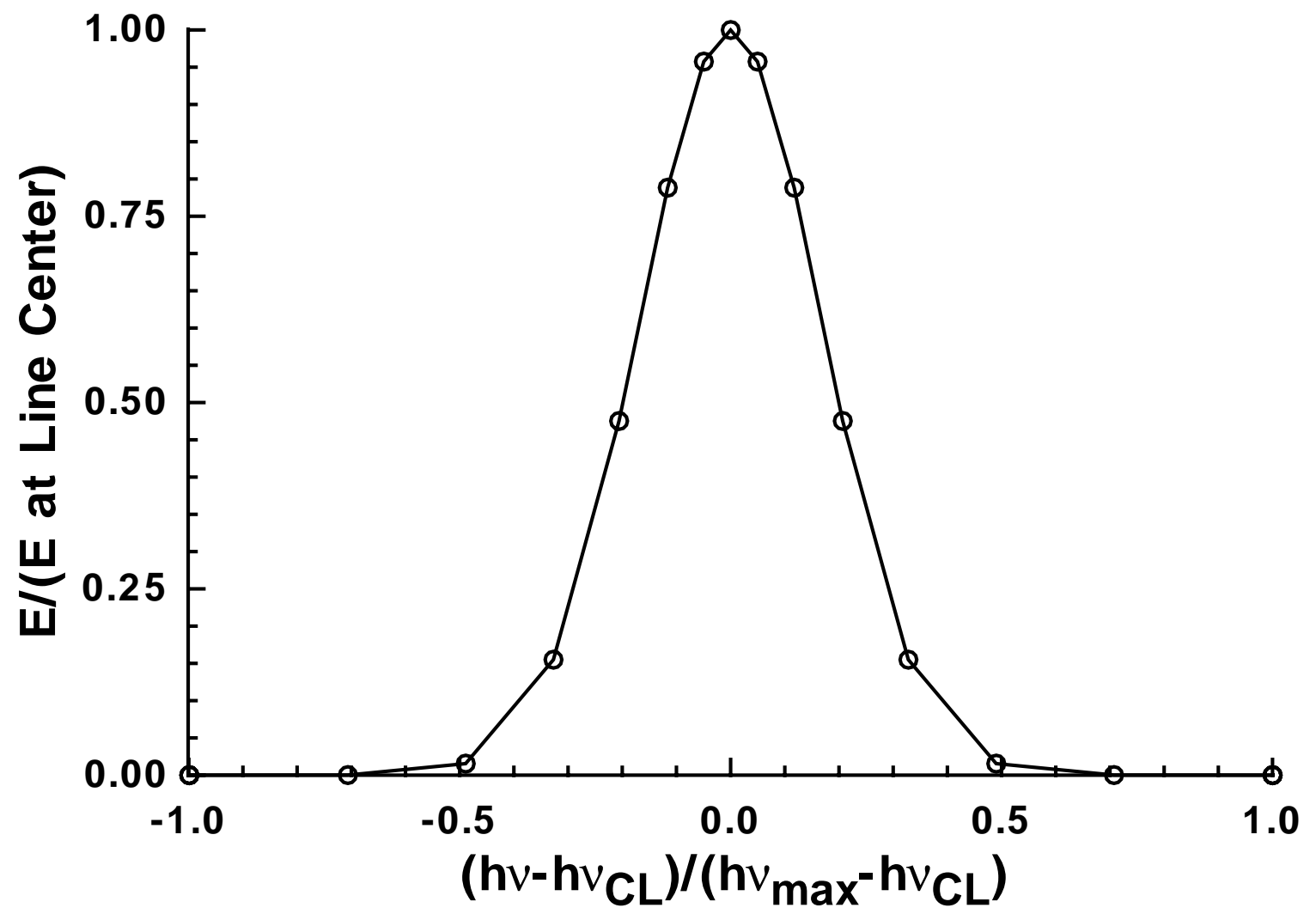

Figure 1: Distribution of Spectral Points for an Atomic Line 


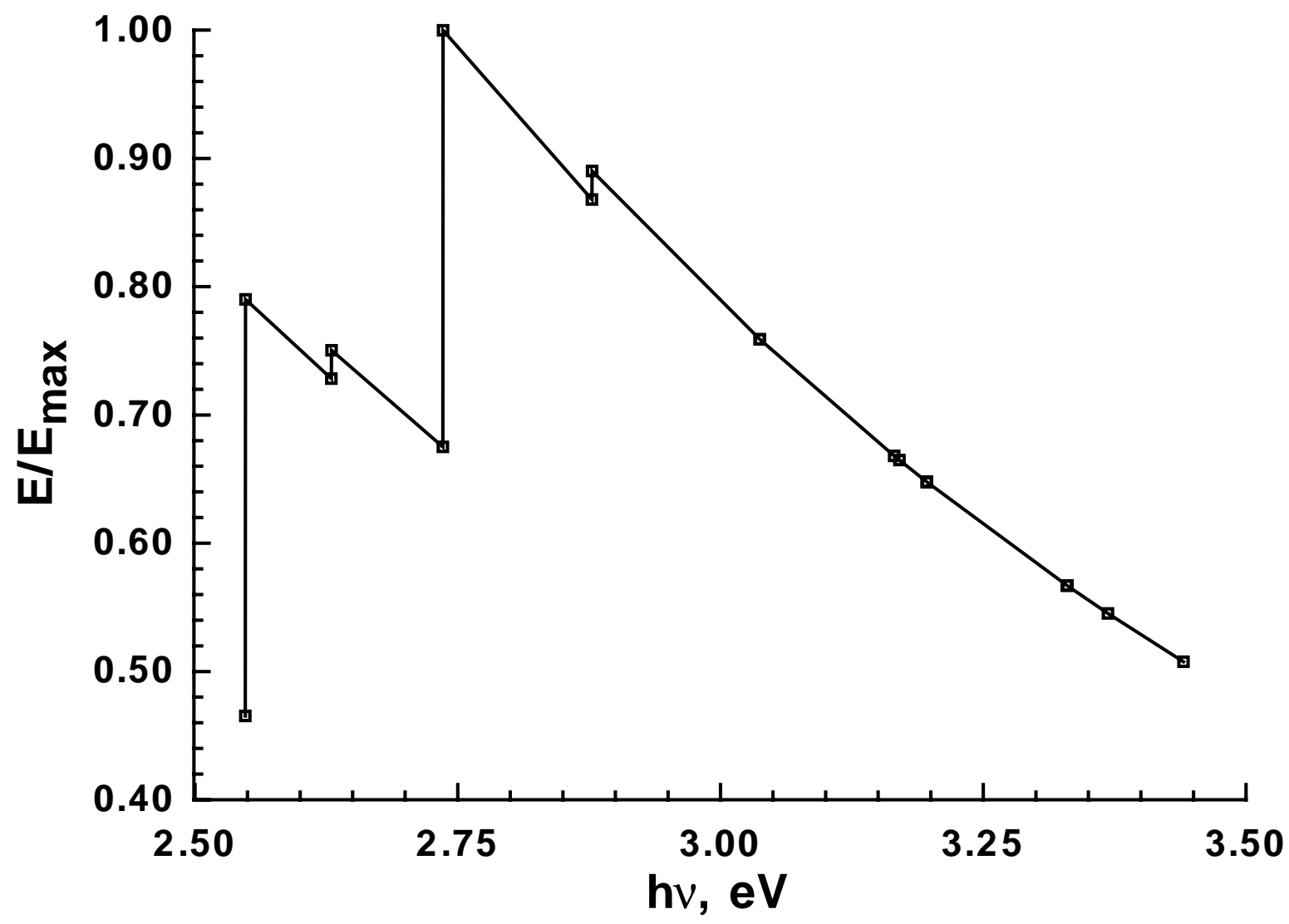

Figure 2: Example of Spectrum Optimization for Bound-Free Continuum 


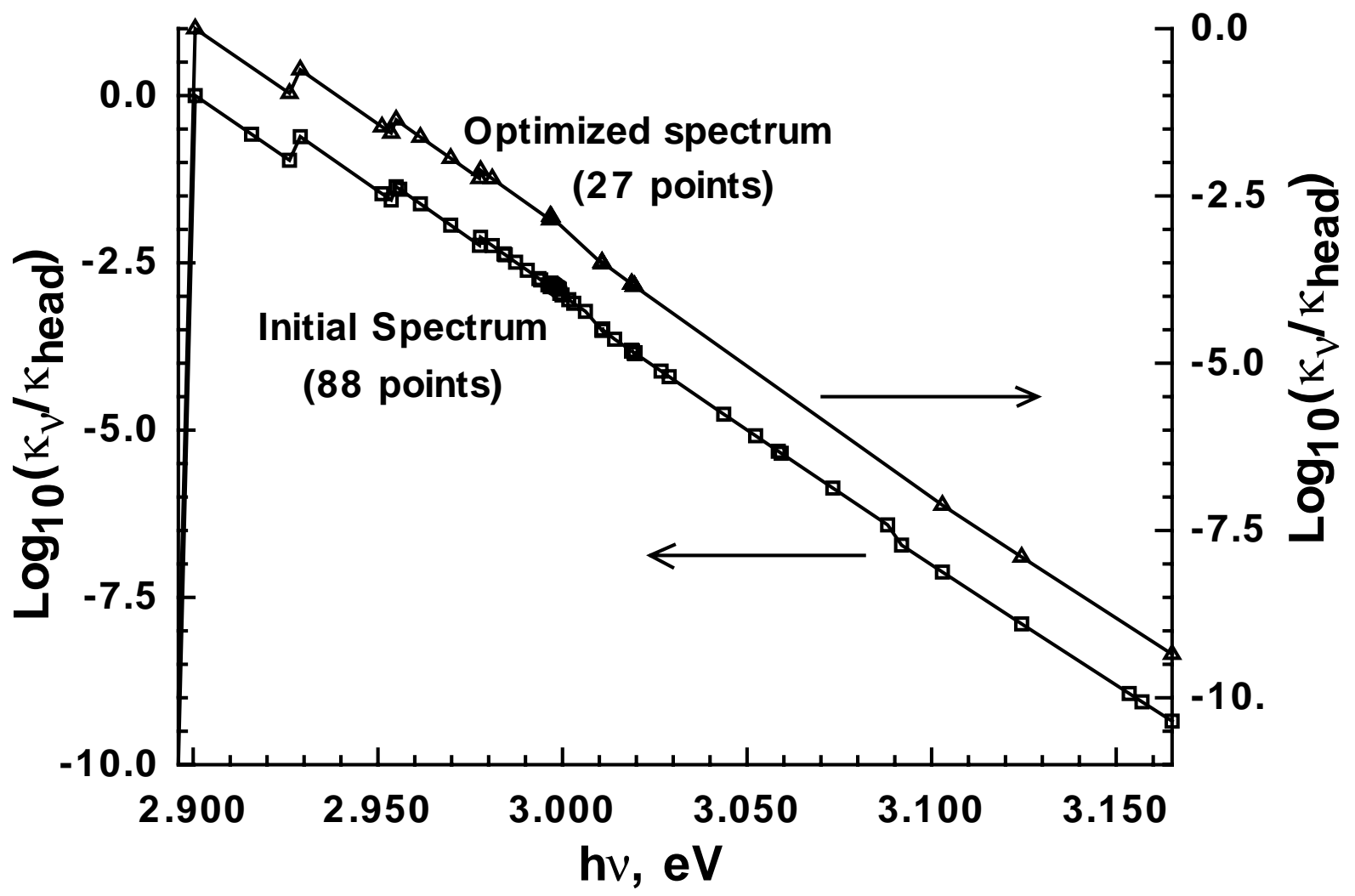

Figure 3: Example of Spectrum Optimization for a Vibrational Band 


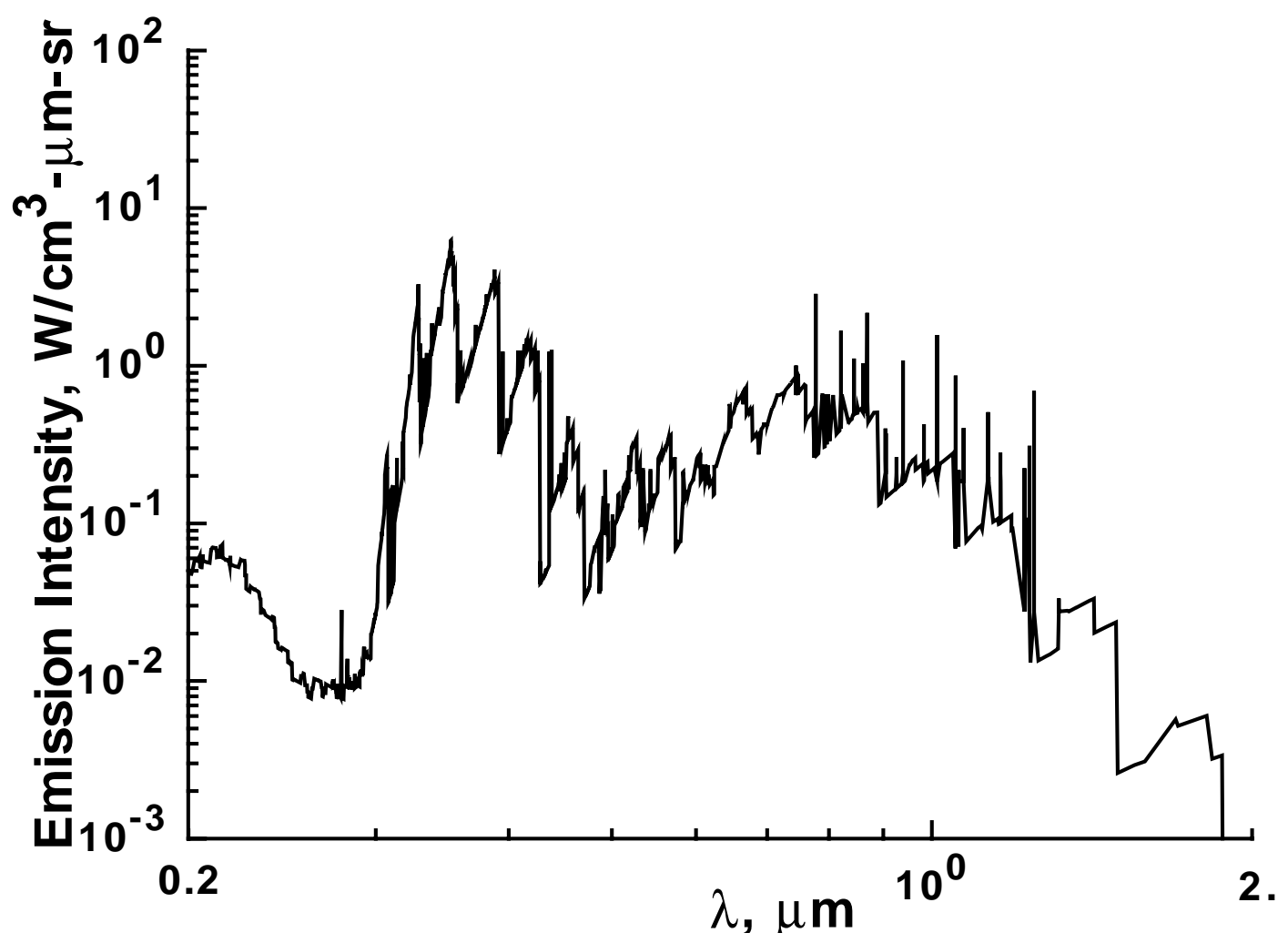

Figure 4: Calculated Spectrum for the Peak Radiation Point of the AVCO Experiment 


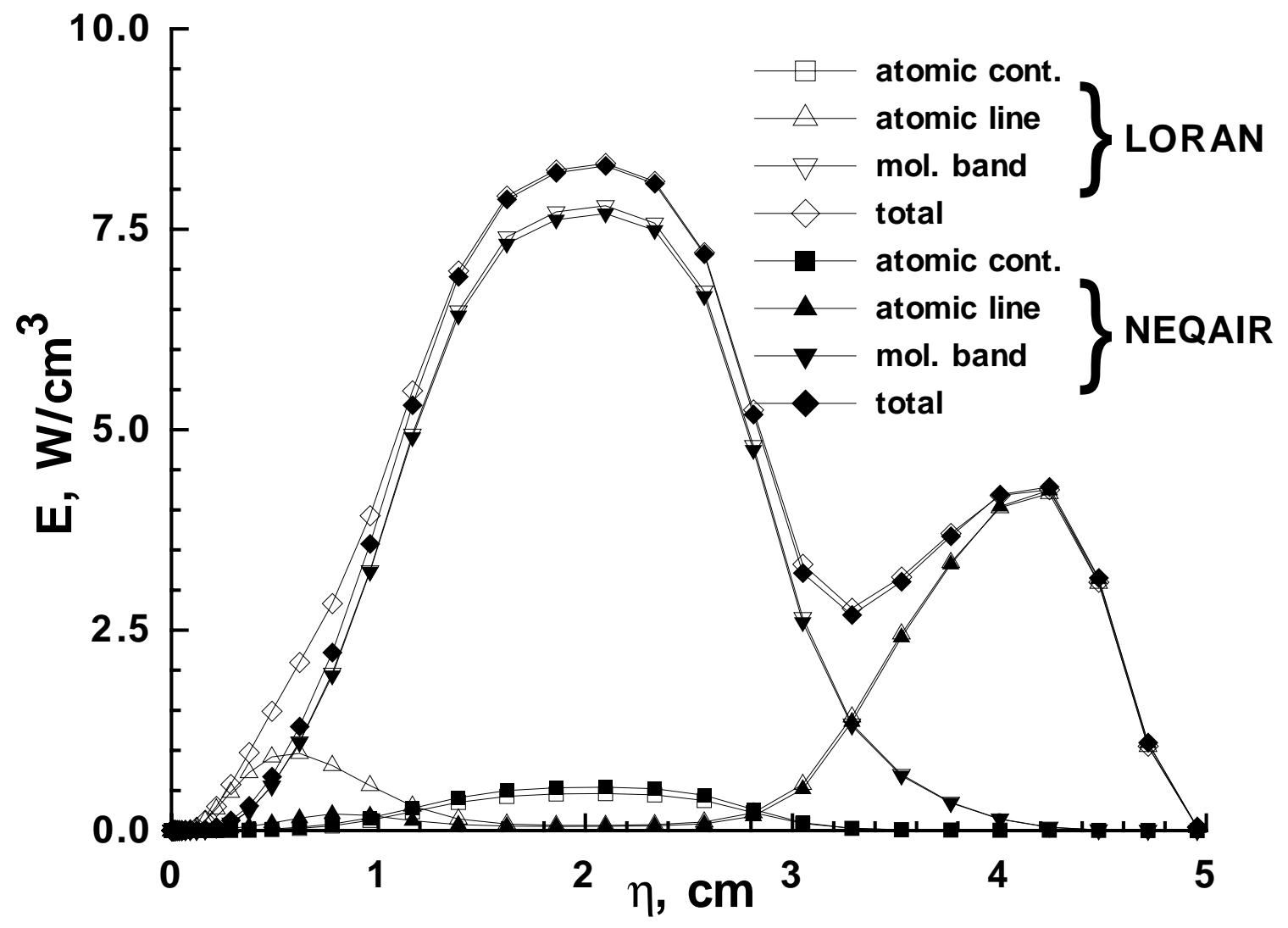

Figure 5: Emission Profiles for FIRE II at $1634 \mathrm{sec}$ with Comparison to NEQAIR 


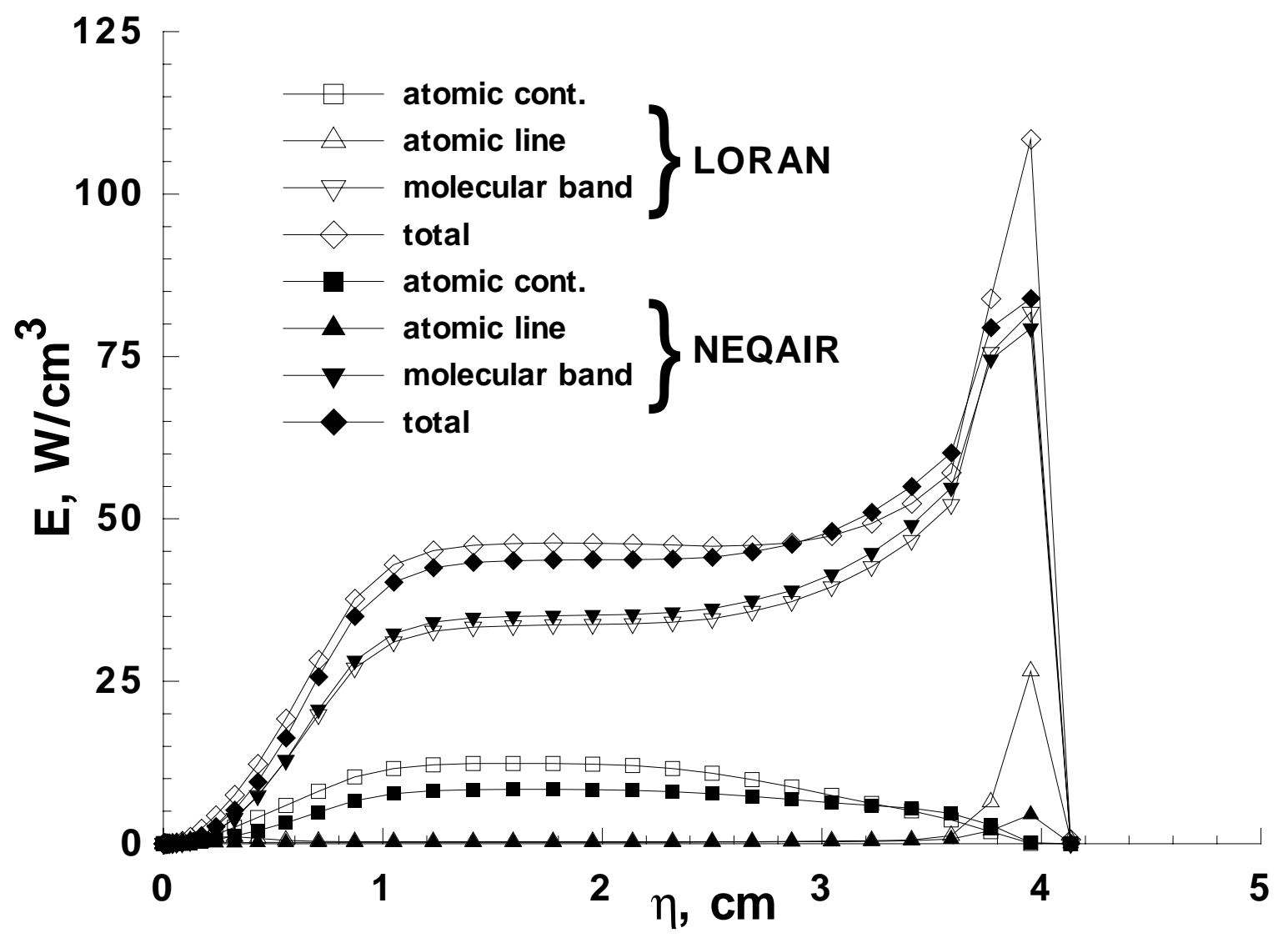

Figure 6: Emission Profiles for FIRE II at 1637.5 sec with Comparison to NEQAIR 


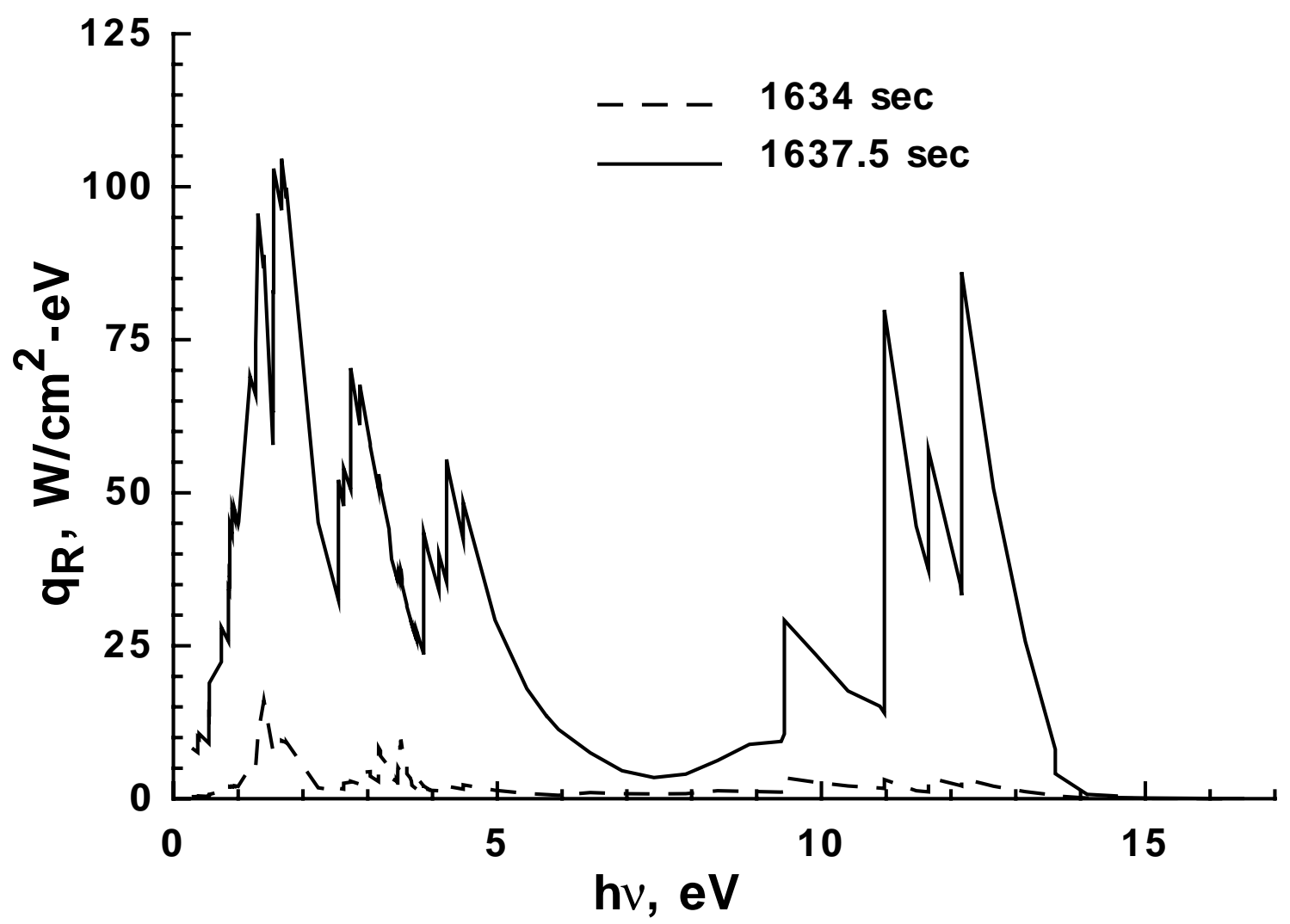

Figure 7: Spectral Variation of Stagnation Point Radiative Heat Transfer for FIRE II 


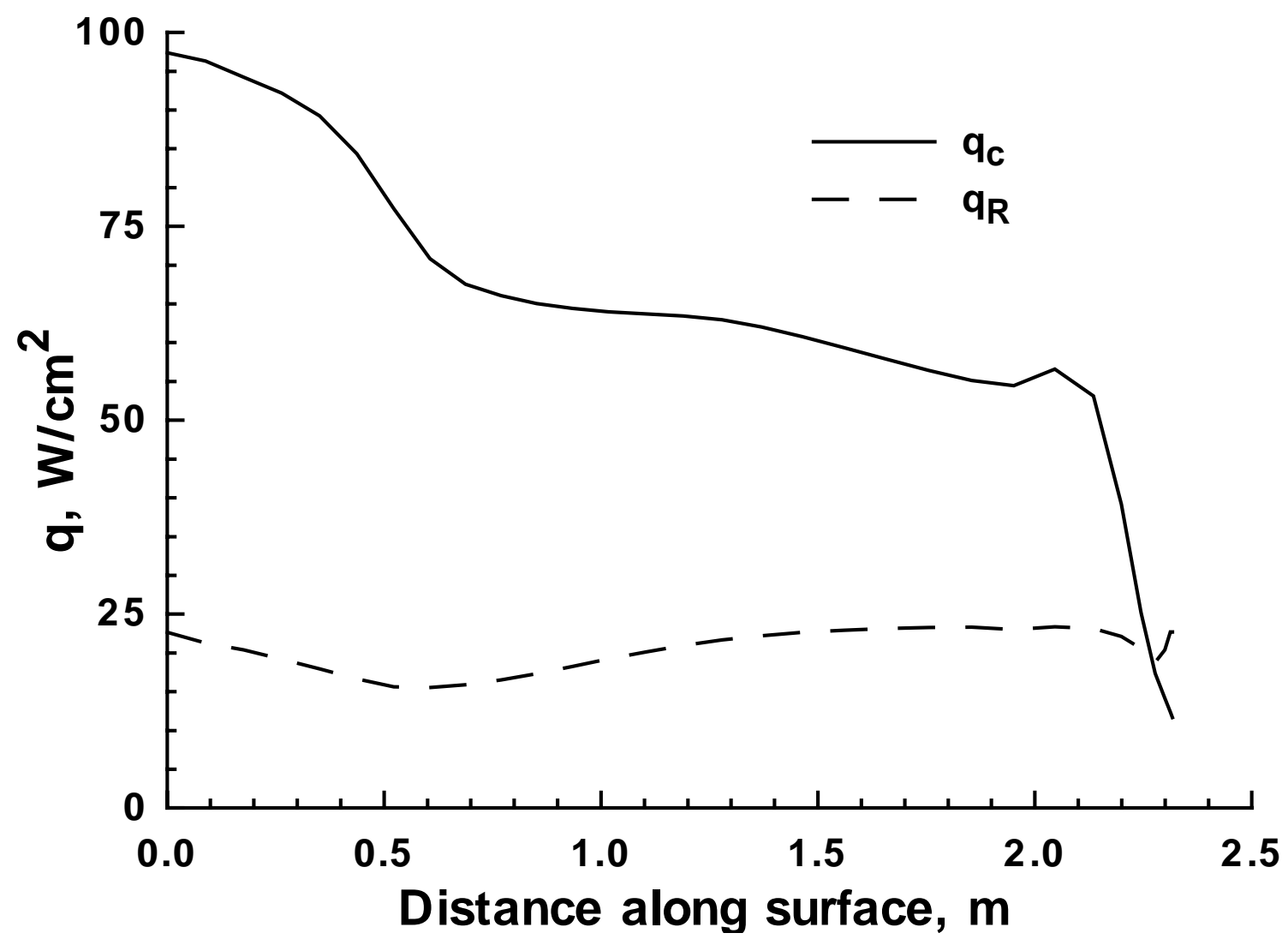

Figure 8: Variation of Convective and Radiative Heat Transfer on a $60^{\circ}$ Sphere Cone with $R_{N}=1.08 \mathrm{~m}, \mathrm{~V}=12 \mathrm{~km} / \mathrm{sec}$ and $\mathrm{H}=80 \mathrm{~km}$ 